Few-Body Systems 0, 1-4 (2019)

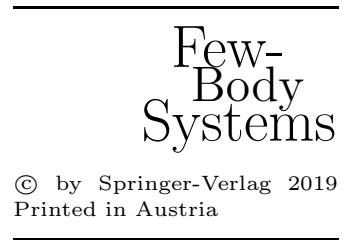

\title{
Two-nucleon problem in semi-relativistic baryon chiral perturbation theory
}

\author{
D. Djukanovic, J. Gegelia*, S. Scherer, M. R. Schindler \\ Institut für Kernphysik, Johannes Gutenberg-Universität, D-55099 Mainz, Germany
}

\begin{abstract}
We consider a symmetry-preserving approach to the nucleonnucleon scattering problem in the framework of the higher-derivative formulation of baryon chiral perturbation theory. Within this framework the leading-order amplitude is calculated by solving renormalizable equations and corrections are taken into account perturbatively.
\end{abstract}

It has been claimed that Weinberg's program for the few-nucleon sector of baryon chiral perturbation theory [1] encounters conceptual problems. The NN potential of the effective field theory (EFT) is non-renormalizable already at leading order (LO). The iteration of the potential in the LS equation generates divergent terms with structures which are not included in the original potential. Therefore, the renormalization of the solution to the LS equation requires the contributions of an infinite number of higher-order counter-terms. It has been argued that the coefficients of the divergent parts of the counter-terms contributing in low-order calculations would set the scale of the corresponding renormalized couplings [2]. Therefore, even if these couplings were natural at some value of the renormalization scale, they would become unnaturally large for slightly different values of this parameter. This problem, in different variations, has been addressed as the inconsistency of Weinberg's approach. On the other hand, cutoff EFT advocates the point of view that the "consistency problem" of Weinberg's approach is irrelevant and one can perform the calculations by suitably choosing the cutoff parameter (see, e.g., [3, 4, [5, 6, 7]). The controversy still remain unresolved.

Here, we shortly present a new approach developed in Ref. [8].

The standard effective Lagrangian leads to UV divergences. These can be handled by considering a nucleon propagator with an improved UV behavior [9]

$$
i S_{F}(p)=\frac{i}{\not p-m+i \epsilon} F\left(L^{2}, \boldsymbol{p}^{2}\right),
$$

*Alternative address: Institute of High Energy Physics, Tbilisi State University, 0186 Tbilisi, Georgia 
where the regulating function $F$ can be chosen as e.g.

$$
F\left(L^{2}, \boldsymbol{p}^{2}\right)=\frac{L^{4 N_{\Psi}}}{\left(L^{2}+\boldsymbol{p}^{2}\right)^{2 N_{\Psi}}} \text { or } F\left(L^{2}, \boldsymbol{p}^{2}\right)=\exp \left\{-2\left(\frac{\boldsymbol{p}^{2}}{L^{2}}\right)^{N_{\Psi}}\right\} .
$$

The equation for the nucleon-nucleon scattering amplitude reads

$$
T=V+V G T
$$

Expanding $T, V$, and $G$ in small parameters (like the pion mass and small momenta),

$$
G=G_{0}+G_{1}+\cdots, V=V_{0}+V_{1}+\cdots, T=T_{0}+T_{1}+\cdots,
$$

and substituting in Eq. (3), we solve $T$ order by order (analogously to the KSW approach [2]). At leading order we obtain

$$
T_{0}=V_{0}+V_{0} G_{0} T_{0}
$$

Here, the LO two-nucleon propagator $G_{0}=-i S_{F}^{(1)} S_{F}^{(1)}$ and $S_{F}^{(1)}$ is defined by

$$
S_{F}(p)=\frac{m F\left(L^{2}, \boldsymbol{p}^{2}\right)\left(1+\gamma^{0}\right) / 2}{\sqrt{\boldsymbol{p}^{2}+m^{2}}\left(p_{0}-\sqrt{\boldsymbol{p}^{2}+m^{2}}++i \epsilon\right)}+\cdots=S_{F}^{(1)}(p)+\cdots .
$$

Using the LO amplitude $T_{0}$, the NLO amplitude $T_{1}$ is calculated. Analogously, $T_{0}$ and $T_{1}$ can be used to calculate the NNLO amplitude $T_{2}$ etc.

The LO equation in the center-of-mass frame reduces to

$$
\mathcal{T}_{0}\left(\boldsymbol{p}^{\prime}, \boldsymbol{p}\right)=\mathcal{V}_{0}\left(\boldsymbol{p}^{\prime}, \boldsymbol{p}\right)-m \int \frac{d^{3} k}{(2 \pi)^{3}} \mathcal{V}_{0}\left(\boldsymbol{p}^{\prime},-\boldsymbol{k}\right) \frac{1}{\boldsymbol{p}^{2}-\boldsymbol{k}^{2}+i \epsilon} \mathcal{T}_{0}(-\boldsymbol{k}, \boldsymbol{p}),
$$

where

$$
\begin{aligned}
\mathcal{T}_{0}\left(\boldsymbol{p}^{\prime}, \boldsymbol{p}\right) & =\frac{\tilde{T}_{0}\left(\boldsymbol{p}^{\prime}, \boldsymbol{p}\right) F\left(L^{2}, \boldsymbol{p}^{\prime 2}\right) F\left(L^{2}, \boldsymbol{p}^{2}\right) m^{2}}{\left[\left(m^{2}+\boldsymbol{p}^{\prime 2}\right)\left(m^{2}+\boldsymbol{p}^{2}\right)\right]^{1 / 2}} \\
\mathcal{V}_{0}\left(\boldsymbol{p}^{\prime}, \boldsymbol{p}\right) & =\frac{\tilde{V}_{0}\left(\boldsymbol{p}^{\prime}, \boldsymbol{p}\right) F\left(L^{2}, \boldsymbol{p}^{\prime 2}\right) F\left(L^{2}, \boldsymbol{p}^{2}\right) m^{2}}{\left[\left(m^{2}+\boldsymbol{p}^{\prime 2}\right)\left(m^{2}+\boldsymbol{p}^{2}\right)\right]^{1 / 2}} .
\end{aligned}
$$

Here, $\tilde{V}_{0}$ is the sum of the standard contact interaction $\left(V_{0, C}\right)$ and one-pion exchange $\left(\tilde{V}_{0, \pi}\right)$ parts of the LO potential [3]. The potential $\mathcal{V}_{0}\left(\boldsymbol{p}^{\prime}, \boldsymbol{p}\right)$ has a milder ultraviolet behavior than $\tilde{V}_{0}\left(\boldsymbol{p}^{\prime}, \boldsymbol{p}\right)$. In the limit $L \rightarrow \infty$ the OPE part of the effective potential $\mathcal{V}_{0}\left(\boldsymbol{p}^{\prime}, \boldsymbol{p}\right)$ generates no divergences in the ${ }^{1} S_{0}$ wave. The ${ }^{3} S_{1}$ wave requires a single counter-term, which is present in our LO potential. For higher partial waves the singular behavior of the potential is screened by the angular momentum barrier and therefore no counter-terms are required. The numerical analysis confirms the above conclusions. The ${ }^{3} P_{0}$ phase shifts calculated for different values of the parameter $L$ are shown in Fig. 1, Different choices of $L$ correspond to different renormalization schemes [8]. By choosing the renormalization scheme properly one can improve the convergence of perturbative 


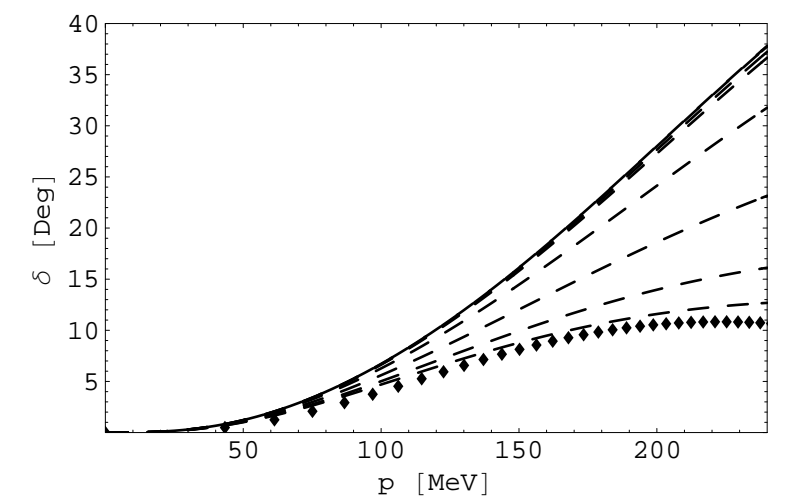

Figure 1. ${ }^{3} P_{0}$ partial wave $n p$ phase shifts for different values of $L$ parameter compared with the data points from the Nijmegen PWA [10]. The curves are shown for $L=750,1000,2000$, $6000,20000,30000,100000,500000(\mathrm{MeV})$, the solid line corresponding to the largest value.

series for physical quantities. The best description of the phase shifts at leading order is obtained for $L \sim 750 \mathrm{MeV}$. Corrections to any finite order can be expressed as a sum of a finite number of Feynman diagrams where $T_{0}$ is also interpreted as $\mathrm{NN}$ vertex. The UV behavior of $T_{0}$ guarantees that all divergences generated in the limit $L \rightarrow \infty$ in corrections at any finite order can be canceled by counter-term contributions also present at the given order. As a result our new approach is free of "consistency problems".

Acknowledgement. D.D., J.G. and M.R.S. acknowledge the support of the Deutsche Forschungsgemeinschaft (SFB 443).

\section{References}

1. S. Weinberg, Phys. Lett. B 251, 288 (1990).

2. D. B. Kaplan, M. J. Savage, and M. B. Wise, Phys. Lett. B 424, 390 (1998).

3. E. Epelbaum, Prog. Part. Nucl. Phys. 57, 654 (2006).

4. G. P. Lepage, arXiv:nucl-th/9706029

5. J. Gegelia, Phys. Lett. B 429, 227 (1998).

6. J. Gegelia, J. Phys. G 25, 1681 (1999).

7. J. Gegelia and S. Scherer, Int. J. Mod. Phys. A 21, 1079 (2006).

8. D. Djukanovic, J. Gegelia, S. Scherer and M. R. Schindler, to appear in Few-Body Systems, arXiv:nucl-th/0609055.

9. D. Djukanovic, M. R. Schindler, J. Gegelia, and S. Scherer, Phys. Rev. D 72, 045002 (2005).

10. V. G. J. Stoks, R. A. M. Klomp, M. C. M. Rentmeester and J. J. de Swart, Phys. Rev. C 48, 792 (1993), http://nn-online.org. 